\title{
Vision Triggers an Experience-Dependent Sensitive Period at the Retinogeniculate Synapse
}

\author{
Bryan M. Hooks and Chinfei Chen \\ Department of Neurology, F. M. Kirby Neurobiology Center, Children's Hospital Boston, and Program in Neuroscience, Harvard Medical School, Boston, \\ Massachusetts 02115
}

In the mammalian visual system, sensory experience is widely thought to sculpt cortical circuits during a precise critical period. In contrast, subcortical regions, such as the thalamus, were thought to develop at earlier ages in a vision-independent manner. Recent studies at the retinogeniculate synapse, however, have demonstrated an influence of vision on the formation of synaptic circuits in the thalamus. In mice, dark rearing from birth does not alter normal developmental maturation of the connection between retina and thalamus. However, deprivation $20 \mathrm{~d}$ after birth [postnatal day 20 (p20)] resulted in dramatic weakening of synaptic strength and an increase in the number of retinal inputs that innervate a thalamic relay neuron. Here, by quantifying changes in synaptic strength and connectivity in response to different time windows of deprivation, we find that several days of vision after eye opening is necessary for triggering experience-dependent plasticity. Shorter periods of visual experience do not permit similar experience-dependent synaptic reorganization. Furthermore, changes in connectivity are rapidly reversible simply by restoring normal vision. However, similar plasticity did not occur when shifting the onset of deprivation to p25. Although synapses still weakened, recruitment of additional retinal inputs no longer occurred. Therefore, synaptic circuits in the visual thalamus are unexpectedly malleable during a late developmental period, after the time when normal synapse elimination and pruning has occurred. This thalamic sensitive period overlaps temporally with experience-dependent changes in the cortex, suggesting that subcortical plasticity may influence cortical responses to sensory experience.

Key words: vision; thalamus; synaptic plasticity; synapse development; sensitive period; experience-dependent plasticity

\section{Introduction}

Neuronal circuits form and refine during well defined developmental periods. In the visual system, critical periods for experience-dependent cortical plasticity are defined for ocular dominance (Hubel and Wiesel, 1970; Fagiolini et al., 1994; Gordon and Stryker, 1996), orientation selectivity (Crair et al., 1998; White et al., 2001), and direction selectivity (Daw and Wyatt, 1976; Li et al., 2006). Cortical synapses are functional before critical period onset and subsequently acquire the ability to remodel after altered visual experience. In contrast, subcortical regions, including thalamus, superior colliculus, and retina, were thought to develop earlier and be less sensitive to sensory experience. Recent studies, however, show that experience influences circuit development and maintenance in the retina (Tian and Copenha-

Received 0ct. 14, 2007; revised March 26, 2008; accepted March 27, 2008.

This work was supported by the Albert J. Ryan Foundation and National Institutes of Health (NIH) Grant F31 NS048630 (B.M.H.); by NIH Grant R01EY013613 and the Pew Scholars Award (C.C.); and by the Children's Hospital Boston Mental Retardation and Developmental Disabilities Research Center (NIH Grant P01HD18655). We thank X Liu, J. Choi, A. Mardinly, B. Carter, M. Fagiolini, G. Corfas, M. Greenberg, C. J. Shatz, and G. Turrigiano for helpful discussions of the experiments and manuscript, as well as A. Joy and W. Blair for technical assistance.

Correspondence should be addressed to Chinfei Chen at the above address. E-mail: chinfei.chen@childrens.harvard.edu.

B. M. Hooks's present address: Janelia Farm Research Campus, Howard Hughes Medical Institute, Ashburn, VA 20147.

DOI:10.1523/JNEUROSCI.4667-07.2008

Copyright $\odot 2008$ Society for Neuroscience $\quad$ 0270-6474/08/284807-11\$15.00/0 gen, 2003), superior colliculus (Lu and Constantine-Paton, 2004; Carrasco et al., 2005), and thalamus (Hooks and Chen, 2006).

In visual thalamus, retinal ganglion cell (RGC) axons segregate into eye-specific regions before eye opening. Sensory experience does not play an initial role in retinogeniculate circuit formation. Instead, spontaneous activity and molecular signals establish an initial retinotopic map (Galli and Maffei, 1988; Shatz and Stryker, 1988; Feldheim et al., 1998; Penn et al., 1998; Pfeiffenberger et al., 2005). After eye-specific segregation is complete, changes in retinogeniculate synaptic function occur over a developmental time spanning eye opening (Chen and Regehr, 2000; Jaubert-Miazza et al., 2005; Ziburkus and Guido, 2006). Specifically, excess retinal afferents to relay cells prune and remaining afferents strengthen. Spontaneous retinal activity, not vision, drives these functional changes (Hooks and Chen, 2006).

Our studies in mice uncovered a late period of experiencedependent retinogeniculate development. Specifically, dark rearing mice from postnatal day 20 (p20), called late dark rearing (late DR), results in recruitment of additional retinal inputs and weakening of existing inputs innervating thalamic relay neurons (Hooks and Chen, 2006). Thus, changes in visual experience alter circuit connectivity. Two possible models may explain our findings. The first possibility is that experience is not required for normal maturation but is needed for maintenance once synapses form. This model is consistent with studies showing that cortical 




Figure 1. Single-fiber amplitude determination. $\boldsymbol{A}$, Left, Raw traces of a pair of failure trials at $+40 \mathrm{mV}$ (black) and $-70 \mathrm{mV}$ (blue). Calibration: 10 pA, $5 \mathrm{~ms}$. Color lines underneath recordings indicate $10 \mathrm{~ms}$ time window over which data were used to compute amplitude histograms in $0.1 \mathrm{pA}$ bins (sampling interval, $20 \mu \mathrm{s}$ ). Right, Histograms of recordings at $-70 \mathrm{mV}$ (inward current) and $+40 \mathrm{mV}$ (outward current). Red $(-70 \mathrm{mV})$ and green $(+40 \mathrm{mV})$ bars are from before stimulation; blue $(-70 \mathrm{mV})$ and black $(+40 \mathrm{mV})$ bars are after stimulation. Substantial overlap occurs for failure trials and is centered around $0 \mathrm{pA}$ with \pm 5 pA of noise. $\boldsymbol{B}, \boldsymbol{C}$, Examples of three raw traces of successful trials $(\boldsymbol{B} \mathbf{1}-\boldsymbol{B} \mathbf{B})$ and average of all successful trials $(\boldsymbol{C})$ interleaved at $-70 \mathrm{mV}$ (blue) and $+40 \mathrm{mV}$ (black). Calibration is the same as in $\boldsymbol{A}$. All trials in $\boldsymbol{B}$ and $\boldsymbol{C}$ were at the same stimulus intensity. In $\boldsymbol{C}$, traces averaged together are overlaid, with the average trace in red and raw traces in blue and black. Presentation of histograms is as in $\boldsymbol{A}$. Note shift of distribution away from $0 \mathrm{pA}$ for successful trials.

orientation selectivity (Crair et al., 1998) and superior colliculus receptive fields (Carrasco et al., 2005) develop normally with binocular deprivation but degrade after prolonged deprivation. However, this fails to explain our finding that chronic dark rearing from birth does not elicit retinogeniculate plasticity. An alternative hypothesis is that visual experience influences synaptic plasticity during a developmental window after eye opening, and vision before this time is necessary to trigger sensitivity to deprivation.

To distinguish between these models, we examined the ages during which we could elicit sensorydependent retinogeniculate plasticity. We show that $>3 \mathrm{~d}$ of vision after eye opening is needed to trigger plasticity during subsequent deprivation. Moreover, changes in synaptic strength and connectivity require $>4 \mathrm{~d}$ of deprivation. Delaying initiation of deprivation until p25 results in weakening of individual afferents similar to late DR, but additional inputs are not recruited. Finally, returning late DR animals to normal lighting rapidly reverses these synaptic changes. Together, these results suggest a distinct developmental period when thalamic circuits can be influenced by sensory experience.

\section{Materials and Methods}

Lateral geniculate nucleus slice preparation. In compliance with the Institutional Animal Care and Use Committee at Children's Hospital Boston, C57BL/6J mice (p9-p32) were anesthetized with isoflurane and decapitated. The brain was removed and placed in a $4^{\circ} \mathrm{C}$ choline-based cutting solution (in mm: $78.3 \mathrm{NaCl}, 23$ $\mathrm{NaHCO}_{3}, 23$ glucose, 33.8 choline chloride, 2.3 $\mathrm{KCl}, 1.1 \mathrm{NaH}_{2} \mathrm{PO}_{3}, 6.4 \mathrm{MgCl}_{2}$, and $0.45 \mathrm{CaCl}_{2}$ ). Lateral geniculate nucleus (LGN) slices (250 $\mu \mathrm{m}$ ) were cut using a sapphire blade (Delaware Diamond Knives, Wilmington, DE) on a vibratome (VT1000S; Leica, Deerfield, IL) as previously described (Chen and Regehr, 2000). Slices were allowed to recover at $31^{\circ} \mathrm{C}$ for $20-25$ min in the choline cutting solution and for 20-35 $\mathrm{min}$ in isotonic saline solution (in $\mathrm{mm}$ : $125 \mathrm{NaCl}, 25 \mathrm{NaHCO}_{3}, 25$ glucose, $2.5 \mathrm{KCl}$, $1.25 \mathrm{NaH}_{2} \mathrm{PO}_{3}, 1 \mathrm{MgCl}_{2}$, and $2 \mathrm{CaCl}_{2}$ ). Oxygenation $\left(\begin{array}{llll}95 \% & \mathrm{O}_{2} / 5 \% & \left.\mathrm{CO}_{2}\right) & \text { was supplied }\end{array}\right.$ continuously.

Electrophysiology. Whole-cell voltage-clamp recordings of thalamic relay neurons from the contralateral monocular region of the dorsal LGN were performed as previously described (Chen and Regehr, 2000). Glass electrodes of 1.2-2.2 MÙ were filled with a CsF-based internal solution (in mм: $35 \mathrm{CsF}, 100 \mathrm{CsCl}, 10$ EGTA, and 10 HEPES, pH adjusted to 7.32 with $\mathrm{CsOH}$ ). D600 (methoxyverapamil hydrochloride; $0.1 \mathrm{~mm}$; Tocris, Ellisville, $\mathrm{MO}$ ) was added to block voltage-gated calcium channels. Recordings were made in saline with $20 \mu \mathrm{M}$ bicuculline, a $\mathrm{GABA}_{\mathrm{A}}$ receptor antagonist (Tocris). Saline-filled glass pipettes were placed up to 1 $\mathrm{mm}$ away in the optic tract, and these stimulating electrodes were moved repeatedly until reaching the location that gave the largest postsynaptic response. Resolving single-fiber inputs is illustrated in Figure 1. Stimulus intensity was reduced to subthreshold and then increased in $0.25 \mu \mathrm{A}$ increments until synaptic responses returned. As seen in Figure 1, small amplitude synaptic currents $(\sim 10 \mathrm{pA})$ can be discerned during several repetitions at the same stimulus intensity. Typically, more than four repetitions were used to confirm the presence of a small input, and the average of successful trials was used for quantification. Single-fiber measurements included a second input from a given cell if it was recruited during incremental increase in stimulus intensity $(0.25 \mu \mathrm{A})$ and clearly resolvable (five times greater in amplitude) from the first input. NMDAR current time course was deter- 
A

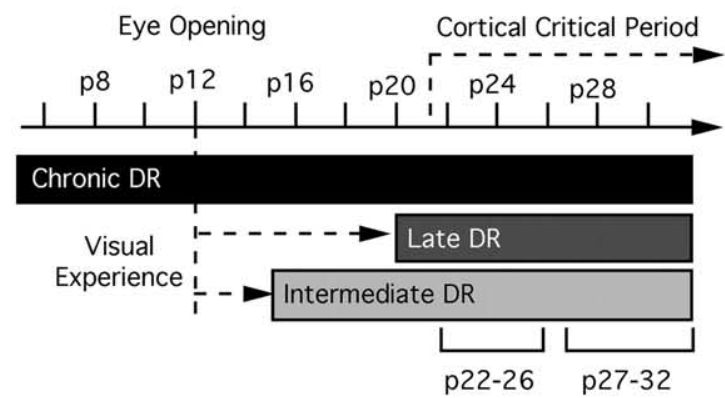

B


C

Stimulus Intensity $(\mu \mathrm{A})$
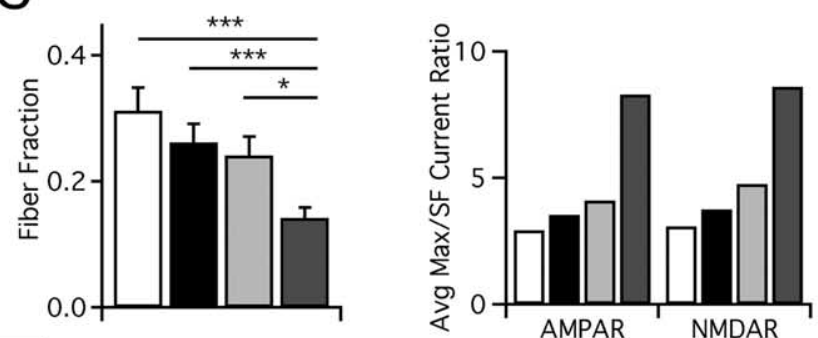

Control

Chronic DR

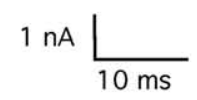

p22 Intermediate DR

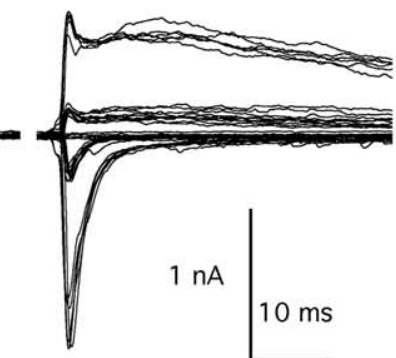

p30 Intermediate DR

p30 Late DR
Late DR

Figure 2. Sensitivity to visual deprivation peaks after a week of visual experience at the retinogeniculate synapse. $\boldsymbol{A}$, Timeline of mouse visual development, annotated with time of eye opening and variation in the amount of visual experience (dotted arrow) or deprivation (black or gray blocks) before evaluation of retinogeniculate synaptic connectivity. The conditions chronic DR (deprivation from $\mathrm{p} 1+$ ), late DR (deprivation from p20+), and intermediate DR (deprivation from $\mathrm{p} 15+$ ) are compared. Ages at which animals were evaluated indicated below the condition. $\boldsymbol{B}$, Recordings from activity-manipulated animals. Top, LGN cell from p22 intermediate DR mouse. Top left, Graph of peak EPSC for AMPAR ( $-70 \mathrm{mV}$; white circles) and NMDAR

mined with a single-exponential fit to the current evoked by maximal stimulation of the optic tract while holding the relay neuron at $+40 \mathrm{mV}$ for $500 \mathrm{~ms}$ after stimulation.

We checked a number of parameters to ensure that the recorded synaptic currents were activated by retinal inputs and not corticothalamic inputs. In the LGN slice preparation, retinal inputs were spatially separated from corticothalamic projections; thus, placement of the stimulating electrode into the optic tract at least $500 \mu \mathrm{m}$ away from the recorded relay neuron makes it unlikely that cortical inputs are activated. In addition, we can distinguish retinal from cortical inputs by their short-term plasticity. Corticothalamic inputs facilitate in response to pairs of stimuli separated by $50 \mathrm{~ms}$, whereas retinal inputs exhibit short-term synaptic depression (Turner and Salt, 1998; Granseth et al., 2002; Reichova and Sherman, 2004). Finally, the AMPAR EPSC decay kinetics of cortical inputs is relatively slower than that of retinal inputs: $>5-6 \mathrm{~ms}$ (Alexander et al., 2006) versus 1.5-3 ms for retinal inputs (Chen and Regehr, 2000). This difference is thought to be, in part, secondary to the location of corticothalamic inputs on distal dendrites, whereas retinal inputs terminate more proximally (Wilson et al., 1984).

Dark rearing. Control animals for dark-reared experiments were raised in microisolator cages in standard animal facility conditions under a $12 \mathrm{~h}$ light/12 h dark cycle. Dark-reared animals were placed in a light-tight container [luminance $<0.02$ lux by Light Probe Meter (Extech Instruments, Waltham, MA)]. In experiments in which deprivation began at p15, the eyes were opened at p12 to ensure a uniform $3 \mathrm{~d}$ of visual experience before dark rearing. In other experiments, eyes were permitted to open naturally, which occurs normally at p12 $\pm 1 \mathrm{~d}$ in the C57BL/6J mouse strain. To minimize light exposure before the animals were killed, experimental dark-reared animals were transferred to the lab in an opaque box. Our data for chronic DR and half the data for late DR are drawn from our previous study (Hooks and Chen, 2006).

Evaluation of synaptic pruning. We quantified the synaptic connectivity of retinogeniculate afferents to LGN relay cells using estimates of number of connected fibers. These numbers are not exact and likely an underestimate because of the limitations inherent to the slice preparation, such as cut or damaged afferents. The first method we used, the fiber fraction (Hooks and Chen, 2006), was calculated as the population average of the ratio of singlefiber current divided by the corresponding maximal current amplitude response obtained from the same cell. Thus, fiber fraction $=\left[\Sigma\left(\right.\right.$ single fiber $_{i} l$ maximal response $\left.\left.e_{i}\right)\right] / N$, where the values of single-fiber and maximal current are obtained from each individual cell $i$ (from 1 to $N$ ), and $N$ is the total number of single-fiber/maximal response pairs obtained from recording from many cells. This method allows us to gather estimates for each cell, making differences in fiber number statistically testable (Hooks and Chen, 2006). Both AMPAR and NMDAR single-fiber data for each cell were used. When inverted, the fiber fraction gives an estimate of the number of connected RGC axons. The second method used, the ratio method, estimates the number of afferents based on the ratio of average single-fiber current to the average maximal current (given as a range of two numbers, one using AMPAR and the other NMDAR values) over a population of neurons in the same age group and condition. The number of fibers estimated using this method is in general agreement with the fiber fraction. We did not attempt to count steps in each recording, because we felt making the distinction between trial-to-trial variation and recruitment of an additional small afferent was arbitrary. We detail our reasons for the selection of this methodology in the supplemental material (available at www.jneurosci.org).

$\leftarrow$

(+40 mV; black circles) plotted against stimulus intensity. Top right, Superimposed traces of EPSCs recorded while increasing stimulus intensity. Middle, LGN cell from $\mathrm{p} 30$ intermediate DR animal. Bottom, Neuron from p30 late DR mouse. C, Estimated connectivity. Left, Fiber fraction is computed as the average of the ratio of each single-fiber input divided by the maximal current for the same cell, as described previously by Hooks and Chen (2006). Significant differences exist between late DR and all other conditions. Right, Ratio of average maximal current to average single-fiber (SF) current for AMPAR and NMDAR. Control: p22-p26, 33 cells from 11 animals; p27-p32, 39 from 13. Chronic DR: p22-p26, 37 from 11; p27-p32, 32 from 13. Intermediate DR: p22-p26, 33 from 10; p27-p32, 41 from 15. Late DR: p27-p32, 63 from 21. * $p<0.05$; ${ }^{* * *} p<0.001$. 



Figure 3. A week of visual experience before deprivation maximizes plasticity at the retinogeniculate synapse. $\boldsymbol{A}$, Top, Analysis of single-fiber AMPAR currents for mature control and deprived mice. Single-fiber AMPAR currents are measured at $-70 \mathrm{mV}$ after manipulations of visual experience. Histograms are divided into bins of $50 \mathrm{pA}$. Note the large number of weak fibers $(<100 \mathrm{pA})$ in all conditions, especially pronounced in late DR, and non-normal distribution. $n=48,45$, and 64 for control, intermediate DR, and late DR, respectively. Bottom, Cumulative probability plots for the same. $B$, Synaptic current after manipulations of visual experience is measured at $-70 \mathrm{mV}$ (AMPAR) and $+40 \mathrm{mV}$ (NMDAR, slow component). The peak current amplitude in response to activation of a single retinal afferent is measured as singlefiber current, and maximal current is measured after excitation of the entire optic tract. Average single-fiber (left) and maximal current (right; note changes in scale) amplitude for AMPAR (top) and NMDAR (bottom) currents assessed in control mice, chronic DR, intermediate DR, and late DR, with ages tested noted below. Control: p22-p26, 33 cells from 11 animals; p27-p32, 35 from 13. Chronic DR: p22-p26, 37 from 11; p27-p32, 39 from 13. Intermediate DR: p22-p26, 33 from 10; p27-p32, 41 from 15. Late DR: p27-p32, 63 from 21. ${ }^{*} p<0.05$; ${ }^{* *} p<0.01$; ${ }^{* * *} p<0.001$.

Statistical analysis. Because our measurements were not distributed normally, a nonparametric Kruskal-Wallis test was used to identify whether or not differences existed between groups in our experiments. To determine whether pairwise differences existed between groups, we used a Dunn post hoc test, which corrected for multiple comparisons (Zar, 1999). When other tests were used, these are specified in the text (e.g., Fisher's exact test).

\section{Results}

A week of visual experience before deprivation is necessary to trigger maximal receptivity to deprivation-induced plasticity We assessed experience-dependent synapse remodeling by quantifying two aspects of the retinogeniculate synapse: single-fiber strength and an estimate of the average number of RGCs that innervate a given relay neuron. Average single-fiber strength was obtained from the peak EPSC amplitude response to minimal stimulation. Figure 1 illustrates how we obtain the single-fiber response. Initially, we stimulate at an intensity that results in failure to evoke synaptic currents at holding potentials of -70 $\mathrm{mV}$ [inward current, black trace, through AMPA receptors (AMPARs)] and $+40 \mathrm{mV}$ [outward current, green trace, through both AMPARs and NMDA receptors (NMDARs)]. Amplitude histograms of the recorded currents during a $10 \mathrm{~ms}$ window before and after the stimulus are plotted on the right. With incremental increases in stimulus intensity, an intensity level is reached that reliably evokes a small synaptic current (Fig. 1 B1$B 3$ ). Figure $1 C$ shows the average single-fiber response (left) and amplitude histogram distribution obtained from three trials each at -70 and $+40 \mathrm{mV}$. Thus, single-fiber synaptic currents that are $<10 \mathrm{pA}$ can be measured (see Materials and Methods for more detail).

After recording the response to minimal stimulation, we increased stimulation intensity gradually to recruit a greater number of afferents in the optic tract and quantified the maximal current response at high stimulation intensity. Both AMPARand NMDAR-mediated responses, recorded at $-70 \mathrm{mV}$ and +40 $\mathrm{mV}$, respectively, were measured. To estimate the number of RGCs that innervate relay neurons, we compared the relative contribution of a single retinal fiber to the maximal synaptic current evoked using much greater optic nerve stimulus intensities. Two related methods are used as measures of this connectivity between RGC and relay neurons: the ratio and fiber fraction methods [see supplemental material (available at www. jneurosci.org) and Hooks and Chen (2006)].

Our previous study revealed that dark rearing mice from p20 for 7-12 d (late DR) resulted in a reduction of the average AMPAR input strength to less than one-half of control and increased the average number of retinal inputs that innervate a relay neuron (Hooks and Chen, 2006). In contrast, a similar change in synaptic connectivity was not observed when mice were chronically dark reared from birth to p27-p32 (chronic DR). One main difference between chronic DR and late DR manipulations is a week-long period of visual experience (from p12 to p20 in late DR mice); thus, we tested whether the length of visual experience influences the synaptic response to visual deprivation. We shortened the length of visual experience before deprivation. Intermediate DR mice, instead, had only $3 \mathrm{~d}$ of sensory experience (from p12 to p15), before they were dark reared (Fig. $2 A)$. We assessed intermediate DR mice at two ages. First, we examined these mice at p22-p26, when the length of deprivation (7-11 d) was similar to late DR. This allowed us to compare the effects of sensory deprivation for a fixed duration of time after $3 \mathrm{~d}$ of visual experience. Second, we studied intermediate DR mice at p27-p32, the same chronological age as late DR mice. This later age range allowed us to assess whether plasticity might occur during a fixed age range and whether prolonged periods of depri- 

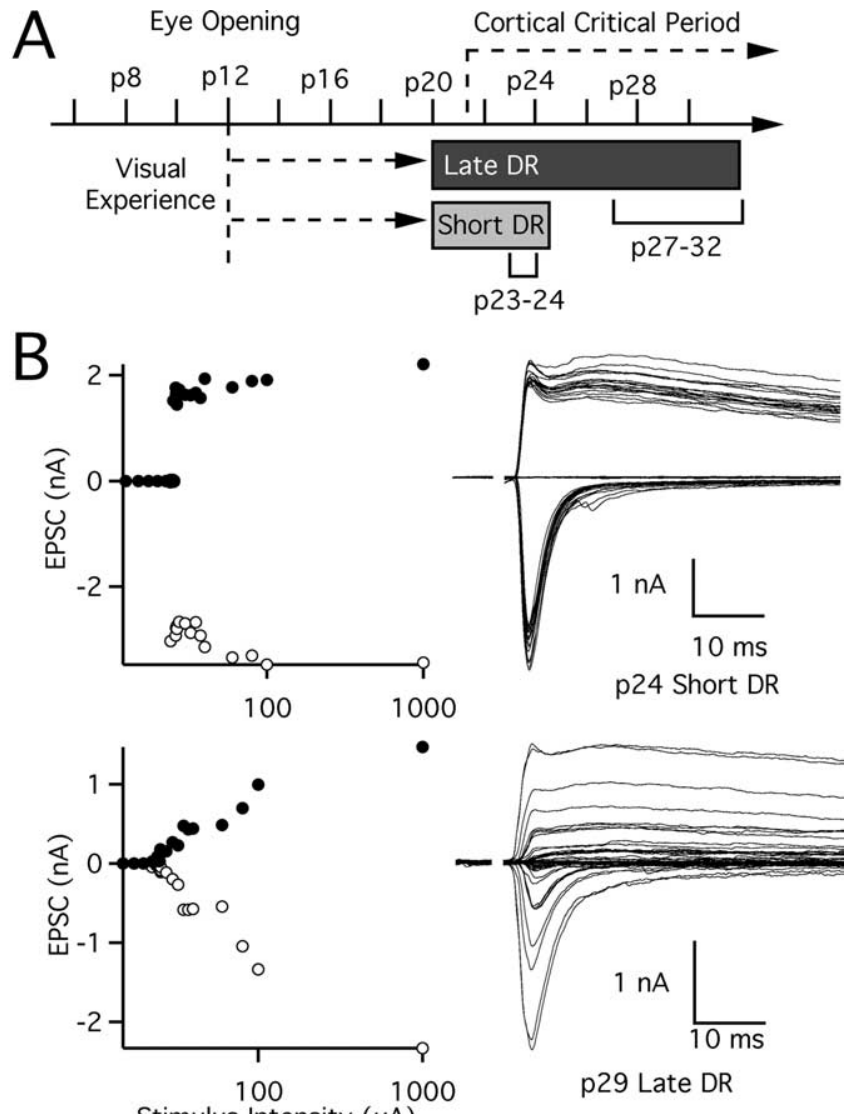

Stimulus Intensity $(\mu \mathrm{A})$

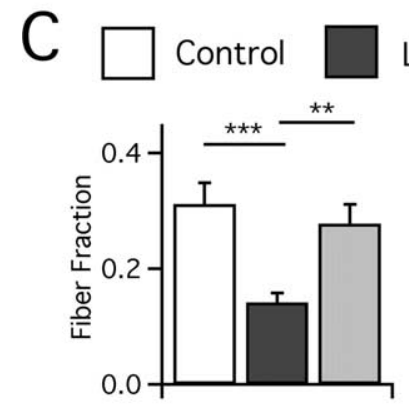

Late DR $\square$ Short DR

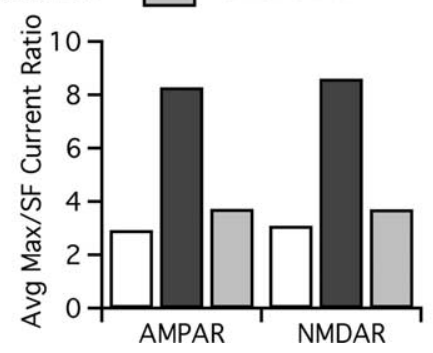

Figure 4. Reorganization of synaptic connectivity requires more than $4 \mathrm{~d}$ of deprivation. $\boldsymbol{A}$, Experimental paradigm showing visual experience and deprivation after dark rearing at p20. Animals evaluated at p23-p24 (short DR) are compared with those at p27-p32 (late DR), where time after deprivation is the only variable. $\boldsymbol{B}$, Recordings from visually deprived animals: p24 short DR (top) and p29 late DR (bottom), presented as in Figure 2. C, Rearrangements in retinal ganglion cell innervation of LGN relay cells occurs after visual deprivation, but such changes take longer than $4 \mathrm{~d}$ to occur. Changes are quantified by both the fiber fraction method (left) and the ratio method (right). Statistical test results are shown for fiber fraction. SF, Single fiber. Control: p27-p32, 39 cells from 13 animals. Late DR: p27-p32, 63 from 21. Short DR: p23-p24, 35 from 15. ${ }^{* *} p<0.01 ;{ }^{* * *} p<0.001$.

vation after vision might uncover more plasticity. Examples of recordings from these conditions are shown in Figure $2 B$.

We found that visual deprivation after a shorter period of visual experience does not induce the same degree of synaptic plasticity as late DR. Single-fiber AMPAR and NMDAR currents, significantly reduced in late DR, are not similarly affected in intermediate DR animals, either after 1 week of deprivation (p22p26) or at later ages (p27-p32; single-fiber AMPAR for intermediate DR vs control, $p>0.2$, Kruskal-Wallis test with post hoc Dunn test) (Fig. 3). Compared with late DR mice at p27-p32, intermediate DR mice exhibit more inputs stronger than $1 \mathrm{nA}$
$(13 / 45$ in intermediate DR; $9 / 64$ in late DR; $p=0.03$ by Fisher's exact test), although the difference of average AMPAR singlefiber strengths is not significant $(p>0.2)$. Plasticity is not entirely absent in these mice, however, because maximal AMPAR currents are increased in a relatively short developmental time. This increase occurs within the first week of deprivation by $\mathrm{p} 22$ $(p<0.001$ vs control at $\mathrm{p} 22-\mathrm{p} 26)$ and persists until maturity (Fig. 3) ( $p<0.05$ vs control at p27-p32). Maximal NMDAR currents are not similarly affected.

Despite an increase in AMPAR maximal current and no change in single-fiber current, estimates from the ratio of the average maximal strength to the average single-fiber strength suggest little change in the number of retinal inputs that innervate relay neurons of intermediate DR animals when compared with that of control animals (average maximal current/singlefiber current ratio of 4-5 for intermediate DR vs 3 for control at p27-p32) (Fig. 2C). In contrast, more retinal fibers are recruited in late DR mice (ratio of $8-9$ ). To make these results statistically testable, we determined the fraction of current that each single fiber contributes to a cell's maximal response for all neurons in a given condition, an index we call fiber fraction. Estimates using the fiber fraction method agree with changes in the ratio method, finding that synaptic connectivity in intermediate DR animals is not significantly different from control animals $(p>0.05)$, whereas a significant number of additional fibers are recruited by late DR $(p<0.05)$ (Fig. $2 C)$. Thus, we conclude that whereas some synaptic properties change with deprivation after $3 \mathrm{~d}$ of vision, a full week of experience is required to induce maximal synaptic reorganization in response to deprivation.

\section{Four days of deprivation is insufficient to induce maximal changes in retinothalamic connectivity}

The time course of retinogeniculate synaptic changes in vivo may provide insight into the underlying mechanisms of synaptic remodeling. For example, long-term potentiation and long-term depression protocols induce robust changes in minutes in vitro, whereas structural changes in the circuitry may take a longer period of time. Therefore, we next examined how rapidly reorganization occurs after visual deprivation. Our previous study showed that changes in synaptic strength and connectivity in response to late DR are maximal by $\mathrm{p} 27$, because we find no difference in fiber strength between animals dark reared from p 20 for $7 \mathrm{~d}$ when compared with those dark reared for 10-12 d (Hooks and Chen, 2006) (see also supplemental Fig. 1, available at www.jneurosci.org as supplemental material). Thus, we tested whether we could detect changes in synaptic properties at a shorter time after the onset of deprivation at p20 (dark rear for 3-4 d, test at p23-p24; short DR) (Fig. $4 A$ ).

We found that synaptic connectivity was not disrupted after 3-4 d of deprivation (Fig. 4). Figure $4 B$ compares examples of recordings made after $4 \mathrm{~d}$ (top; p24) and $9 \mathrm{~d}$ (bottom; p29) of deprivation. The amplitude of the first single fiber recruited in the p24 animal (top) is quite substantial, and subsequent increases in optic tract stimulation recruit little additional current. The average fiber fraction for short DR is $0.28 \pm$ $0.03 \%$, compared with $0.31 \pm 0.04 \%$ and $0.14 \pm 0.02 \%$ for control $(p>0.05)$ and late DR $(p<0.002)$, respectively. We therefore conclude that, although some cellular and molecular changes may be induced during the first $3 \mathrm{~d}$ of deprivation, afferent number is not significantly altered. Thus, the full effects of late visual deprivation on retinogeniculate connectivity take longer to occur. 




Figure 5. Weakening of retinogeniculate synapses requires more than $4 \mathrm{~d}$ of deprivation. $A$, Single-fiber AMPAR currents for control, late DR, and short DR mice presented in histograms as before. $n=48,64$, and 45 for control, late DR, and short DR. Cumulative probability plot (below) given for these three conditions. $\boldsymbol{B}$, Bar graphs of synaptic current at $-70 \mathrm{mV}$ (AMPAR) and $+40 \mathrm{mV}$ (NMDAR, slow component). Average single-fiber (left) and maximal current (right; note changes in scale) amplitude for AMPAR (top) and NMDAR (bottom) currents was assessed in control and late DR mice at p27-p32 and in short DR mice at p23-p24. Control: p27-p32, 39 cells from 13 animals. Late DR: p27-p32, 63 from 21. Short DR: p23-p24, 35 from 15. ${ }^{*} p<0.05 ;{ }^{* *} p<0.01$.

When compared with control mice at p27-p32, we found that deprivation for 3-4 d after p20 also did not significantly change single-fiber current amplitudes. The distribution of single-fiber AMPAR currents is nearly identical to control (Fig. 5A) $(p>$ $0.05)$, although it is also not significantly different from that of late DR animals (Fig. 5) ( $p>0.05$ ). Moreover, maximal AMPAR and NMDAR currents are also not affected by short DR (Fig. 5) ( $p>0.05$ vs control).

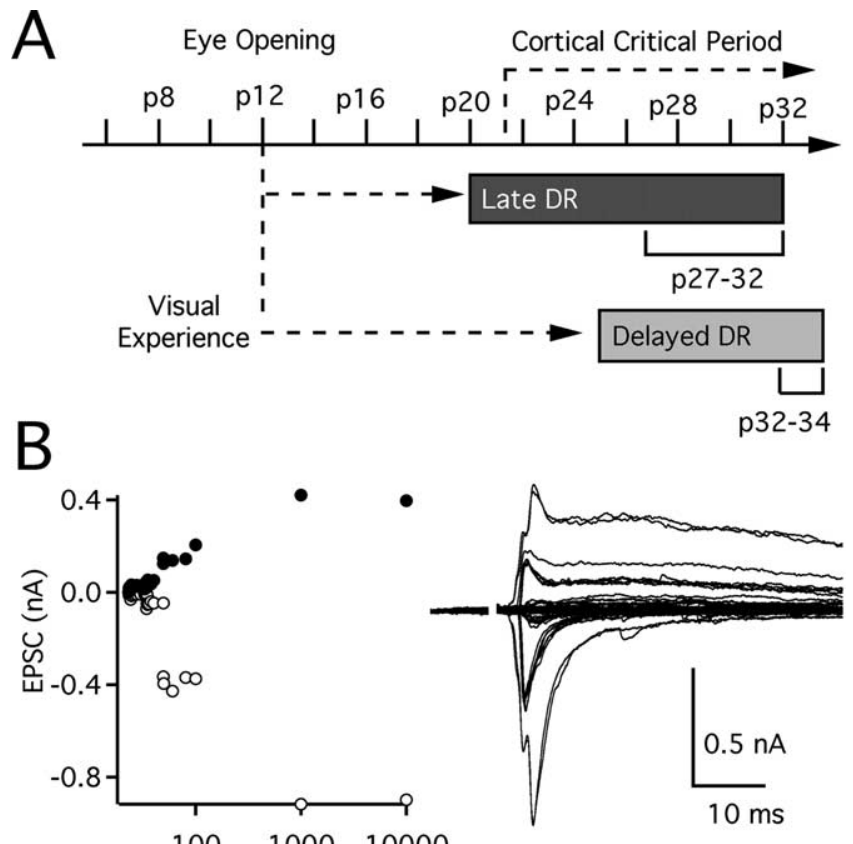

timulus Intensity $(\mu \mathrm{A})$

p33 Delayed DR


Figure 6. Visual deprivation at later ages does not recruit additional retinogeniculate afferents. $\boldsymbol{A}$, Timeline of late DR and delayed DR (deprivation from p25+) experimental paradigms, with ages evaluated at bottom. $\boldsymbol{B}$, Recording from p33 delayed DR animal: graph of peak EPSC amplitudes for all stimulus intensities (left) and traces at $-70 \mathrm{mV}$ and $+40 \mathrm{mV}$ (inward and outward current, respectively; right). C, Left, Estimated connectivity assessed by fiber fraction as described previously. Control: p27-p32, 39 cells from 13 animals. Late DR: p27-p32, 63 from 21. Delayed DR: p32-p34, 32 from 12. Significant differences are annotated as ${ }^{*} p<0.05$ and ${ }^{* *} p<0.001$. Right, Ratio of average maximal current to average singlefiber current for AMPAR and NMDAR. SF, Single fiber.

The characteristics of plasticity change when deprivation is initiated at a later age

Our intermediate DR experiments suggest that the onset of a retinogeniculate plasticity occurs after $>3 \mathrm{~d}$ of vision. We next explored whether this form of plasticity persists at later ages or whether plasticity occurs during a distinct sensitive period, by delaying the initiation of dark rearing until p25 (delayed DR) (Fig. 6A). Thus, animals received normal visual experience until p25. After a week of visual deprivation, we evaluated synaptic strength and connectivity in p32-p34 mice. Because it required a week of visual deprivation to achieve the complete effects of late DR, we gave delayed DR animals a similar period of deprivation. An example recording from a p33 delayed DR mouse is shown in Figure $6 \mathrm{~B}$. These results were then compared with control and late DR animals at p27-p32, an age range when developmental changes in single-fiber and maximal current strength have pla- 


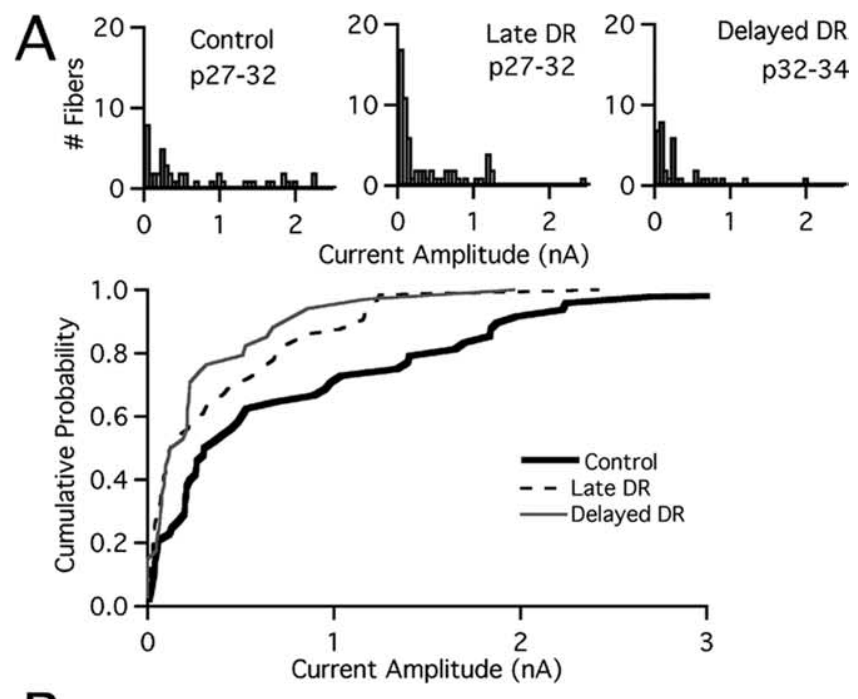

B
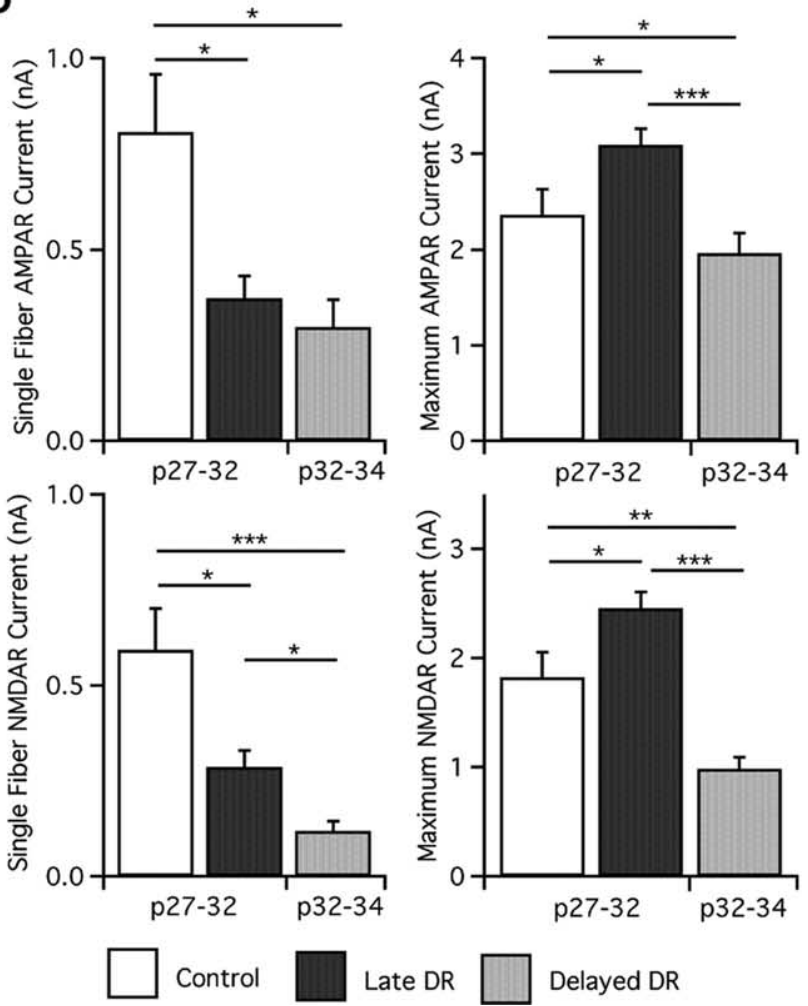

Figure 7. Visual deprivation at later ages results in synaptic weakening. $\boldsymbol{A}$, Top, Analysis of single-fiber AMPAR currents at $-70 \mathrm{mV}$ for mature control and deprived mice. Histograms are divided into bins of $50 \mathrm{pA}$. Note that the large number of weak fibers $(<100 \mathrm{pA})$ is pronounced in late DR and delayed DR. $n=48,64$, and 34 for control, late DR, and delayed DR, respectively. Bottom, Cumulative probability plots for the same. $\boldsymbol{B}$, Synaptic current after manipulations of visual experience is measured at $-70 \mathrm{mV}$ (AMPAR) and $+40 \mathrm{mV}$ (NMDAR, slow component). Average single-fiber (left) and maximal current (right, note changes in scale) amplitude for AMPAR (top) and NMDAR (bottom) currents assessed in control mice, late DR, and delayed DR. Control: p27-p32, 39 cells from 13 animals. Late DR: p27-p32, 63 from 21. Delayed DR: p32p34, 32 from 12. ${ }^{*} p<0.05 ;{ }^{* *} p<0.01 ;{ }^{* * *} p<0.001$.

teaued. Therefore, we compared similar periods of deprivation, with a slight offset in the chronological age of the animals.

We found that deprivation from p25 results in a change of synaptic strength, similar to late DR. Single-fiber AMPAR currents in delayed DR are significantly smaller than control animals (Fig. 7) ( $p<0.05$, Kruskal-Wallis test with post hoc Dunn test), and of similar magnitude to late DR mice. Furthermore, singlefiber NMDAR currents are smaller than either control $(p<$ $0.001)$ or late DR $(p<0.05)$ mice. However, maximal currents were not similarly affected as in late DR. Indeed, maximal AMPAR currents are significantly smaller than both late DR $(p<$ $0.001)$ and control $(p<0.05)$. Maximal NMDAR currents show an identical pattern. Thus, although deprivation at both ages can induce plasticity in the strength of individual afferents, the overall character of experience-dependent changes is not identical.

Because delayed DR reduced both single-fiber and maximal current amplitudes relative to control mice, we inferred that numbers of connected afferents might be unchanged. To test this, we computed the fiber fraction for these conditions (Fig. 6). Our results reveal that, whereas late DR recruits a number of additional fibers, significantly fewer afferents are recruited in delayed DR $(p<0.05)$. The delayed DR fiber fraction was not significantly different from control mice. Thus, we find that although visual deprivation at p25 induces weakening of individual afferents, synaptic remodeling is significantly different from that of late DR, because the number of retinal afferents innervating a given relay neuron does not change.

\section{Light exposure reverses deprivation-induced plasticity}

The results from our studies suggest that there is a late developmental sensitive period of plasticity during which the connectivity between retinal ganglion cells and thalamic relay neurons can be changed. To test whether sensory experience-dependent plasticity of synaptic circuits is reversible, animals were deprived from p20 until p27 and then returned to a standard light cycle (DR recovery) (Fig. 8A). After at least $3 \mathrm{~d}$ of renewed visual experience, recordings were made from p30-p32 LGN relay cells and compared with that of late DR animals at p27-p32. This comparison was appropriate because the effects of late DR are maximal by age p27 (supplemental Fig. 1, available at www. jneurosci.org as supplemental material). Recordings after DR recovery appeared strikingly similar to control recordings (Fig. $8 B$, top). Significant changes in synaptic strength and connectivity were evident. Fiber fraction increased, consistent with a significant reduction in the number of connected fibers in DR recovery versus late $\mathrm{DR}(p<0.001)$. The number of retinal inputs that innervate a relay neuron in DR recovery is no different from that in p27-p32 control mice ( $p>0.05$, DR recovery vs control) (Fig. $8 C$ ). Thus, the deprivation-induced increase in neuronal connections can be undone rapidly, showing that the retinogeniculate connection remains malleable past $\mathrm{p} 27$.

The changes in synaptic connectivity were accompanied by changes in the strength of individual afferents. Single-fiber AMPAR currents strengthened in DR recovery $(p<0.02$ vs late DR) and were comparable in amplitude to those in control mice. Amplitude histograms in Figure 9 show the elimination of a large number of weak $(<100 \mathrm{pA})$ afferents in DR recovery: 3 of 30 fibers are weak $(<100 \mathrm{pA})$ in DR recovery versus 21 of 61 weak afferents in late DR ( $p=0.011$, Fisher exact). Elimination of a large number of small, weak afferents is notable, because even in our control mice, $>20 \%$ of the afferents remain weak at p27-p32. Similarly, single-fiber NMDAR currents are strengthened after $\mathrm{DR}$ recovery $(p<0.02$ vs late $\mathrm{DR})$. On the other hand, maximal currents do not completely reverse within $3 \mathrm{~d}$. Average maximal AMPAR currents are not significantly different from either control or late DR, consistent with incomplete reversal. Thus, we conclude that both deprivation-induced changes in strength and connectivity at the retinogeniculate synapse can be undone rapidly by return to normal visual experience. This demonstrates that a 



Figure 8. Deprivation-induced changes in retinogeniculate connectivity can be rapidly reversed by restoration of normal vision. $\boldsymbol{A}$, Timeline showing periods of visual experience and deprivation after dark rearing at p20. Animals are evaluated at p27-p32 (late DR) or p30-p32 (DR recovery) after allowing a short period of reexposure to normal vision. $\boldsymbol{B}$, Recordings from visually manipulated animals: p32 DR recovery (top) and p27 late DR (bottom). $C$, Reversal of changes in retinogeniculate connectivity in visual thalamus after restoration of normal sensory experience. Connectivity of retinogeniculate afferents is estimated by fiber fraction method (left) and ratio (right) methods. SF, Single fiber. Control: p27-p32, 39 cells from 13 animals. Late DR: p27-p32, 63 from 21. DR recovery: p30-p32, 29 from 18. ${ }^{* * *} p<0.001$.

form of in vivo plasticity of synaptic circuits exists in visual thalamus until at least p27.

\section{Discussion}

Our studies show experience-dependent plasticity at the retinogeniculate synapse that occurs long after onset of vision. Even after substantial pruning from $\sim 10$ weak inputs to $3-4$ stronger inputs shortly after eye opening, the potential for resculpting synaptic circuits remains late in development. Late deprivation results in recruitment of many small inputs and weakening of existing inputs, changing circuit connectivity. However, trigger-
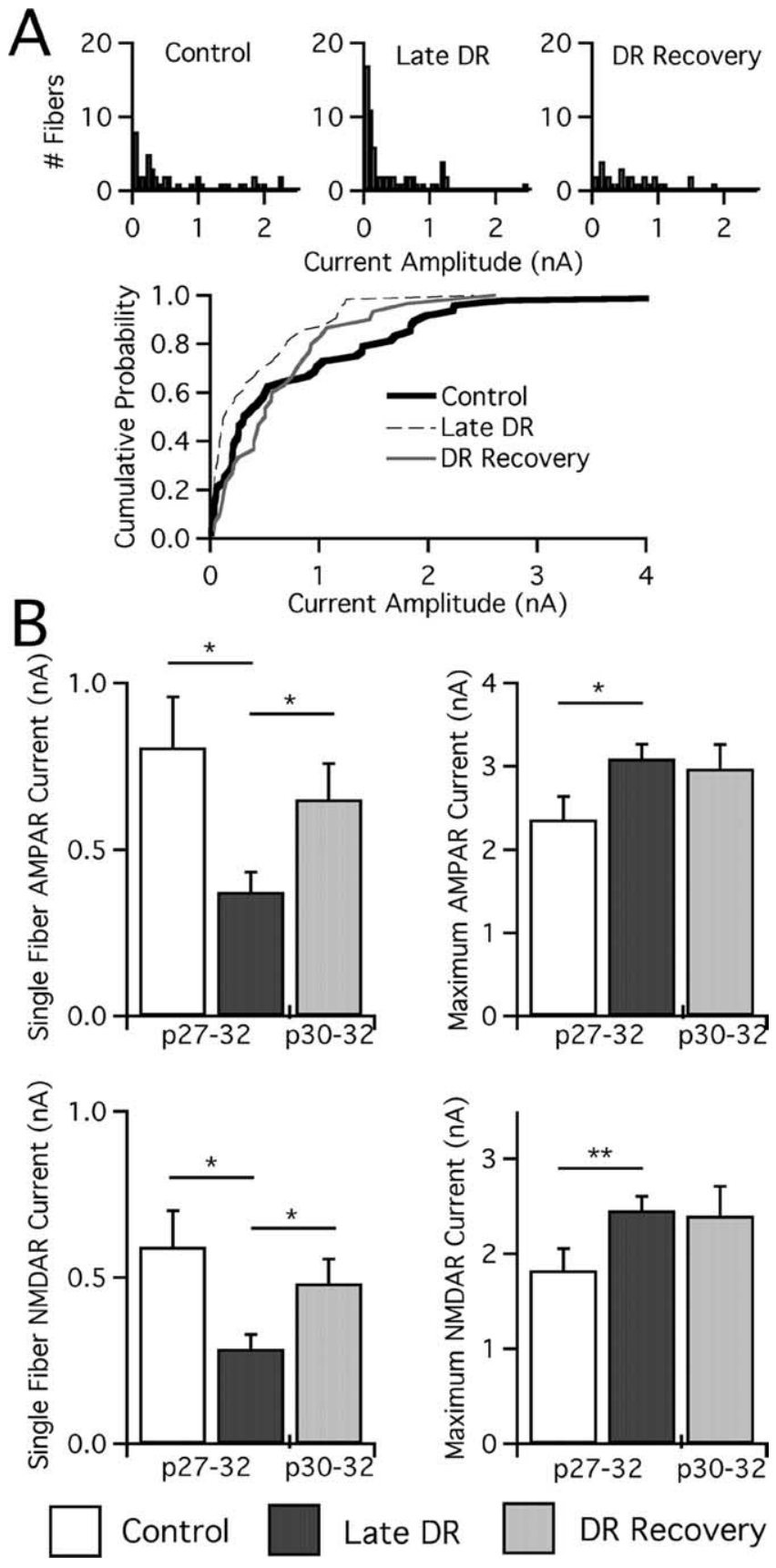

Figure 9. Restoration of normal vision strengthens retinogeniculate afferents. $\boldsymbol{A}$, Top, Single-fiber AMPAR currents measured at $-70 \mathrm{mV}$ for mature control, late $D R$, and DR recovery mice are plotted as histograms in bins of $50 \mathrm{pA}$. Note changes in the number of small afferents $(<100 \mathrm{pA})$, especially for late DR and DR recovery. $n=48,64$, and 30 for control, late DR, and $D R$ recovery. $A$, Bottom, Cumulative probability plots comparing the same three conditions. $\boldsymbol{B}$, Measurements of synaptic current at $-70 \mathrm{mV}$ (AMPAR) and $+40 \mathrm{mV}$ (NMDAR, slow component), noting significant changes in single-fiber AMPAR and NMDAR current amplitudes after DR recovery. Average single-fiber (left) and maximal current (right; note changes in scale) amplitude for AMPAR (top) and NMDAR (bottom) currents assessed in the oldest age groups (noted below) of control mice, late DR, and DR recovery. Control: p22-p26, 33 cells from 11 animals; p27-p32, 39 from 13. Late DR: p27-p32, 63 from 21. DR recovery: p30 - p32, 29 from 18. ${ }^{*} p<0.05 ;{ }^{* *} p<0.01$.

ing plasticity requires previous visual experience, because chronic and intermediate DR animals do not demonstrate equivalent plasticity. The ability to recruit additional inputs in response to deprivation diminishes by $\mathrm{p} 25$, although reduction in afferent strength still occurs in delayed DR mice. Reexposure to 
light after the recruitment of weak inputs (DR recovery) allows renewed circuit refinement. Whether the connections stabilized on the second round of refinement are the same as those during initial remodeling is unknown. This opens the possibility that synaptic circuits can be rewired during a sensitive period of thalamic development.

\section{Vision triggers thalamic sensitivity to sensory changes}

Our studies suggest that vision-sensitive plasticity at the retinogeniculate synapse can be triggered by dark rearing after $>3 \mathrm{~d}$ of visual experience. Thus, synaptic connections in thalamus are not simply dependent on sensory activity for establishment or maintenance once eyes open. Rather, vision is required to set the stage for later retinogeniculate plasticity. These results are consistent with a model in which the onset of visual experience initiates expression of a particular genetic program that permits plasticity within visual system neurons (Prasad et al., 2002; Majdan and Shatz, 2006; Tropea et al., 2006). Without triggering of this genetic program, as in chronic deprivation, a different set of genes is expressed that prohibit synaptic adaptation to the external environment. Presently we cannot distinguish whether a cumulative amount of vision ( $>3 \mathrm{~d}$ after $\mathrm{p} 12$ ) versus vision at a precise age (such as p18) is necessary to activate experience-dependent plasticity. Future experiments varying the time of eye opening will help discriminate between these possibilities and will better define when and how rapidly sensory-dependent plasticity is triggered.

\section{Effects of dark rearing on receptive field development}

What is the physiological consequence of deprivation-induced changes in strength and connectivity? Additional recruited retinal fibers may make LGN receptive fields (RFs) larger. Alternatively, weakened afferents may be unable to drive relay neuron firing, resulting in smaller RFs. Previous studies in several subcortical regions examining the effects of chronic deprivation on RF properties show mixed results. Prolonged dark rearing in rodents disrupts connectivity, changing receptive fields in superior colliculus (Carrasco et al., 2005). Dark rearing also disrupts ON/ OFF segregation in mouse retina (Tian and Copenhagen, 2003). Consistent with this, LGN receptive field maps in dark-reared ferrets show abnormal ON/OFF responses (Akerman et al., 2002). In vivo recordings show that, consistent with the absence of plasticity we observed in chronic DR, dark rearing kittens from before eye opening causes little LGN RF plasticity (Kalil, 1978; Kratz et al., 1979; Mower et al., 1981). Greater visual cortical plasticity is found during chronic deprivation in rats, causing deficits in RF size, acuity, and orientation selectivity (Fagiolini et al., 1994). In cat, binocular deprivation (Wiesel and Hubel, 1965) and dark rearing (Timney et al., 1978) resulted in reduced visual acuity and cortical cells that were unresponsive or had poorly defined receptive field responses.

Few studies, however, examine the effects of dark rearing after a period of visual experience following eye opening. In late DR experiments, we infer that the recruitment of additional afferents may disrupt normal RF size in LGN. Moreover, changes in LGN responses could potentially alter RF properties of cortical neurons. It would be interesting to study the relative changes of LGN and cortical receptive fields in both late DR and chronic DR mice, because chronic DR did not show the same degree of synaptic reorganization in $\mathrm{LGN}$.

\section{Different forms of experience-dependent plasticity in the visual system}

The best-studied form of experience-dependent plasticity in the visual system is ocular dominance (OD) plasticity in the cortex in response to monocular deprivation (MD) (Hubel and Wiesel, 1970; Fagiolini et al., 1994; Gordon and Stryker, 1996). Comparison between experience-dependent plasticity in the LGN and cortex, however, comes with the caveat that the features of monocular deprivation and dark rearing are quite distinct (Sherman and Spear, 1982). Nevertheless, it is worth comparing some characteristics of the plasticity induced at different regions of the visual system. The kinetics of change in response to dark rearing in the thalamus is slower compared with cortical OD plasticity. Three to four days of MD in mouse result in OD shifts (Gordon and Stryker, 1996; Frenkel and Bear, 2004). Robust changes in intracortical circuitry are equally rapid (Hensch et al., 1998; Hensch, 2005). Similarly, deprivation-induced changes in monocular visual cortex, including changes in synaptic weight and connectivity, can be induced rapidly in rodents (Maffei et al., 2004, 2006), as well as homeostatic changes in quantal amplitude after inhibition of visual activity (Desai et al., 2002). In contrast, we find that large-scale changes in retinogeniculate connectivity take $>4 \mathrm{~d}$ of sensory deprivation.

Reversal of plasticity in LGN, however, occurs at a faster rate than onset of plasticity. The mechanisms underlying these changes in thalamus are still not clear. Anatomical changes in retinal axon arbors underlying thalamic plasticity may take a long time to occur. For example, anatomical changes in thalamocortical arbors occur after a week of monocular deprivation in cat (Antonini and Stryker, 1993), and anatomical changes in rodents are similarly delayed (Antonini et al., 1999) compared with the timing of physiological shifts in OD (Trachtenberg et al., 2000). Although the onset of plasticity may be slow, possibly reflecting the time for the structural reorganization of axon arbors, anatomical remnants of previously induced plasticity could result in more rapid changes during DR recovery (Linkenhoker et al., 2005; Hofer et al., 2006). In the cortex, dendrites are in close proximity to many potential partners but form synapses with only a subset (Shepherd et al., 2005), thus allowing the possibility for rapid connectivity changes without gross changes in arbors. A similar mechanism may provide a structural basis for rapid changes in connectivity during restoration of normal vision.

Our finding in delayed DR experiments shows that the type of plasticity expressed in response to visual deprivation depends on the age and previous experience of the animal. It is possible to eliminate excess afferents and strengthen remaining ones during DR recovery as late as p 32 , yet by $\mathrm{p} 25$ it is more difficult to recruit additional afferents by deprivation. This finding is reminiscent of activity manipulations in cultured hippocampal neurons (Burrone et al., 2002), in which the number of functional inputs to a cell is more dependent on activity earlier in synapse development. Similarly, the expression of visual cortical plasticity in young mice is different than in older mice (Desai et al., 2002; Frenkel and Bear, 2004; Maffei et al., 2004, 2006). Delayed DR, then, does not show that plasticity no longer exists, but that the mechanism of synaptic remodeling is different. That changes in synaptic strength are dissociable from changes in the number of afferent fibers suggests that these features of synaptic circuits are regulated by distinct mechanisms during different developmental time windows. 


\section{Interaction between thalamic and cortical plasticity}

Notably, onset of the retinogeniculate sensitive period overlaps temporally with experience-dependent plasticity in the rodent visual cortex, including ocular dominance plasticity (Fagiolini et al., 1994; Gordon and Stryker, 1996), development of synaptic function (Maffei et al., 2006; Crozier et al., 2007), and regulation of gene expression (Castren et al., 1992; Majdan and Shatz, 2006; Tropea et al., 2006). However, changes in retinogeniculate connectivity cannot directly account for ocular dominance shifts. In mouse, LGN eye-specific segregation is complete by p8 (Godement et al., 1984; Muir-Robinson et al., 2002) and does not desegregate after late DR (data not shown). Our recordings are made in a layer of the LGN that receives innervation exclusively from contralateral eye; thus, both newly recruited afferents in response to late DR and existing ones come from the RGCs in the same eye. We do not believe OD shifts can be explained by the thalamic plasticity we describe. However, recruitment of additional afferents could influence thalamocortical output and cortical responses, including changes in receptive field size and shape. Thus, our results indicate that changes in thalamic connectivity should be considered when interpreting long-term changes in cortex in response to visual manipulations.

Conversely, feedback excitation from cortex may play a role in triggering changes at the retinogeniculate synapse, either developmentally by determining the degree of strengthening and pruning, or acutely by shaping the receptive field in the mature circuit. Thus, future examination of thalamic plasticity in animals in which the cortical critical period is specifically disrupted, including manipulation of inhibitory circuit development (Hensch et al., 1998; Fagiolini et al., 2004), will be interesting. As the role of cortical feedback to thalamus is not completely understood, testing thalamic plasticity in the absence of cortical feedback may aid our understanding of the interplay between cortex and thalamus in sensory perception.

\section{References}

Akerman CJ, Smyth D, Thompson ID (2002) Visual experience before eyeopening and the development of the retinogeniculate pathway. Neuron 36:869-879.

Alexander GM, Fisher TL, Godwin DW (2006) Differential response dynamics of corticothalamic glutamatergic synapses in the lateral geniculate nucleus and thalamic reticular nucleus. Neuroscience 137:367-372.

Antonini A, Stryker MP (1993) Rapid remodeling of axonal arbors in the visual cortex. Science 260:1819-1821.

Antonini A, Fagiolini M, Stryker MP (1999) Anatomical correlates of functional plasticity in mouse visual cortex. J Neurosci 19:4388-4406.

Burrone J, O’Byrne M, Murthy VN (2002) Multiple forms of synaptic plasticity triggered by selective suppression of activity in individual neurons. Nature 420:414-418.

Carrasco MM, Razak KA, Pallas SL (2005) Visual experience is necessary for maintenance but not development of receptive fields in superior colliculus. J Neurophysiol 94:1962-1970.

Castren E, Zafra F, Thoenen H, Lindholm D (1992) Light regulates expression of brain-derived neurotrophic factor mRNA in rat visual cortex. Proc Natl Acad Sci USA 89:9444-9448.

Chen C, Regehr WG (2000) Developmental remodeling of the retinogeniculate synapse. Neuron 28:955-966.

Crair MC, Gillespie DC, Stryker MP (1998) The role of visual experience in the development of columns in cat visual cortex. Science 279:566-570.

Crozier RA, Wang Y, Liu CH, Bear MF (2007) Deprivation-induced synaptic depression by distinct mechanisms in different layers of mouse visual cortex. Proc Natl Acad Sci USA 104:1383-1388.

Daw NW, Wyatt HJ (1976) Kittens reared in a unidirectional environment: evidence for a critical period. J Physiol (Lond) 257:155-170.

Desai NS, Cudmore RH, Nelson SB, Turrigiano GG (2002) Critical periods for experience-dependent synaptic scaling in visual cortex. Nat Neuroscience 5:783-789.
Fagiolini M, Pizzorusso T, Berardi N, Domenici L, Maffei L (1994) Functional postnatal development of the rat primary visual cortex and the role of visual experience: dark rearing and monocular deprivation. Vision Res 34:709-720.

Fagiolini M, Fritschy JM, Low K, Mohler H, Rudolph U, Hensch TK (2004) Specific GABAA circuits for visual cortical plasticity. Science 303:1681-1683.

Feldheim DA, Vanderhaeghen P, Hansen MJ, Frisen J, Lu Q, Barbacid M, Flanagan JG (1998) Topographic guidance labels in a sensory projection to the forebrain. Neuron 21:1303-1313.

Frenkel MY, Bear MF (2004) How monocular deprivation shifts ocular dominance in visual cortex of young mice. Neuron 44:917-923.

Galli L, Maffei L (1988) Spontaneous impulse activity of rat retinal ganglion cells in prenatal life. Science 242:90-91.

Godement P, Salaun J, Imbert M (1984) Prenatal and postnatal development of retinogeniculate and retinocollicular projections in the mouse. J Comp Neurol 230:552-575.

Gordon JA, Stryker MP (1996) Experience-dependent plasticity of binocular responses in the primary visual cortex of the mouse. J Neurosci 16:3274-3286.

Granseth B, Ahlstrand E, Lindstrom S (2002) Paired pulse facilitation of corticogeniculate EPSCs in the dorsal lateral geniculate nucleus of the rat investigated in vitro. J Physiol (Lond) 544:477-486.

Hensch TK (2005) Critical period plasticity in local cortical circuits. Nat Rev Neurosci 6:877-888.

Hensch TK, Fagiolini M, Mataga N, Stryker MP, Baekkeskov S, Kash SF (1998) Local GABA circuit control of experience-dependent plasticity in developing visual cortex. Science 282:1504-1508.

Hofer SB, Mrsic-Flogel TD, Bonhoeffer T, Hubener M (2006) Prior experience enhances plasticity in adult visual cortex. Nat Neurosci 9:127-132.

Hooks BM, Chen C (2006) Distinct roles for spontaneous and visual activity in remodeling of the retinogeniculate synapse. Neuron 52:281-291.

Hubel DH, Wiesel TN (1970) The period of susceptibility to the physiological effects of unilateral eye closure in kittens. J Physiol (Lond) 206:419-436.

Jaubert-Miazza L, Green E, Lo FS, Bui K, Mills J, Guido W (2005) Structural and functional composition of the developing retinogeniculate pathway in the mouse. Vis Neurosci 22:661-676.

Kalil R (1978) Dark rearing in the cat: effects on visuomotor behavior and cell growth in the dorsal lateral geniculate nucleus. J Comp Neurol 178:451-467.

Kratz KE, Sherman SM, Kalil R (1979) Lateral geniculate nucleus in darkreared cats: loss of Y cells without changes in cell size. Science 203:1353-1355.

Li Y, Fitzpatrick D, White LE (2006) The development of direction selectivity in ferret visual cortex requires early visual experience. Nat Neurosci 9:676-681.

Linkenhoker BA, von der Ohe CG, Knudsen EI (2005) Anatomical traces of juvenile learning in the auditory system of adult barn owls. Nat Neurosci 8:93-98.

Lu W, Constantine-Paton M (2004) Eye opening rapidly induces synaptic potentiation and refinement. Neuron 43:237-249.

Maffei A, Nelson SB, Turrigiano GG (2004) Selective reconfiguration of layer 4 visual cortical circuitry by visual deprivation. Nat Neurosci 7:1353-1359.

Maffei A, Nataraj K, Nelson SB, Turrigiano GG (2006) Potentiation of cortical inhibition by visual deprivation. Nature 443:81-84.

Majdan M, Shatz CJ (2006) Effects of visual experience on activitydependent gene regulation in cortex. Nat Neurosci 9:650-659.

Mower GD, Burchfiel JL, Duffy FH (1981) The effects of dark-rearing on the development and plasticity of the lateral geniculate nucleus. Brain Res 227:418-424.

Muir-Robinson G, Hwang BJ, Feller MB (2002) Retinogeniculate axons undergo eye-specific segregation in the absence of eye-specific layers. J Neurosci 22:5259-5264.

Penn AA, Riquelme PA, Feller MB, Shatz CJ (1998) Competition in retinogeniculate patterning driven by spontaneous activity. Science 279:2108-2112.

Pfeiffenberger C, Cutforth T, Woods G, Yamada J, Renteria RC, Copenhagen DR, Flanagan JG, Feldheim DA (2005) Ephrin-As and neural activity are required for eye-specific patterning during retinogeniculate mapping. Nat Neurosci 8:1022-1027. 
Prasad SS, Kojic LZ, Li P, Mitchell DE, Hachisuka A, Sawada J, Gu Q, Cynader MS (2002) Gene expression patterns during enhanced periods of visual cortex plasticity. Neuroscience 111:35-45.

Reichova I, Sherman SM (2004) Somatosensory corticothalamic projections: distinguishing drivers from modulators. J Neurophysiol 92:2185-2197.

Shatz CJ, Stryker MP (1988) Prenatal tetrodotoxin infusion blocks segregation of retinogeniculate afferents. Science 242:87-89.

Shepherd GM, Stepanyants A, Bureau I, Chklovskii D, Svoboda K (2005) Geometric and functional organization of cortical circuits. Nat Neurosci 8:782-790.

Sherman SM, Spear PD (1982) Organization of visual pathways in normal and visually deprived cats. Physiol Rev 62:738-855.

Tian N, Copenhagen DR (2003) Visual stimulation is required for refinement of ON and OFF pathways in postnatal retina. Neuron 39:85-96.

Timney B, Mitchell DE, Giffin F (1978) The development of vision in cats after extended periods of dark-rearing. Exp Brain Res 31:547-560.

Trachtenberg JT, Trepel C, Stryker MP (2000) Rapid extragranular plasticity in the absence of thalamocortical plasticity in the developing primary visual cortex. Science 287:2029-2032.
Tropea D, Kreiman G, Lyckman A, Mukherjee S, Yu H, Horng S, Sur M (2006) Gene expression changes and molecular pathways mediating activity-dependent plasticity in visual cortex. Nat Neurosci 9:660-668.

Turner JP, Salt TE (1998) Characterization of sensory and corticothalamic excitatory inputs to rat thalamocortical neurones in vitro. J Physiol (Lond) 510:829-843.

White LE, Coppola DM, Fitzpatrick D (2001) The contribution of sensory experience to the maturation of orientation selectivity in ferret visual cortex. Nature 411:1049-1052.

Wiesel TN, Hubel DH (1965) Comparison of the effects of unilateral and bilateral eye closure on cortical unit responses in kittens. J Neurophysiol 28:1029-1040.

Wilson JR, Friedlander MJ, Sherman SM (1984) Fine structural morphology of identified X-and Y-cells in the cat's lateral geniculate nucleus. Proc R Soc Lond B Biol Sci 221:411-436.

Zar JH (1999) Biostatistical analysis, Ed 4. New York: Prentice Hall.

Ziburkus J, Guido W (2006) Loss of binocular responses and reduced retinal convergence during the period of retinogeniculate axon segregation. J Neurophysiol 96:2775-2784. 